\title{
ABORDAGEM FISIOTERAPÊUTICA NA DOR PÓS-OPERATÓRIA: A ELETROESTIMULAÇÃO NERVOSA TRANSCUTÂNEA (ENT)
}

\section{PHISICALTHERAPY APPROACHING IN POSTOPERATIVE PAIN: TRANSCUTANEOUS ELECTRIC NERVE STIMULATION (TENS)}

\section{Cristine Homsi Jorge Ferreira ${ }^{1}$; Ana Carolina Sartorato Beleza ${ }^{2}$}

\section{INTRODUÇÃO}

A dor e o seu alívio representam uma preocupação para a humanidade desde sempre. $\mathrm{O}$ fenômeno doloroso é de natureza subjetiva, e a observação da manifestação dolorosa é uma das principais portas de acesso que os profissionais de saúde podem ter a essa subjetividade.

Mesmo com todo o progresso da medicina visando desvendar, interpretar e tratar o fenômeno doloroso, ele ainda é hoje inadequadamente entendido, provavelmente por ser sensação individual, única e exclusiva de cada ser, difícil de caracterizar e generalizar'.

Compreender e avaliar a dor possibilita não só sua melhor caracterização e de sua possível doença de base, mas também aproxima o terapeuta do paciente, tornando o profissional mais atento para entender o que a dor significa e os malefícios que causa para o paciente, favorecendo assim o estabelecimento da terapêutica mais eficiente.

Segundo o Comitê de Taxonomia, da Associação Internacional para o Estudo da Dor (International Association for Study of Pain), ela é definida como "uma experiência emocional e sensorial desagradável, associada a lesões reais ou potenciais, ou descrita em termos dessas lesões" (IASP - Subcomitee on Taxonomy, 1986) ${ }^{2}$. Melzack e Wall descrevem a dor como experiência pessoal e subjetiva, influenciada pelo aprendizado cultural, significado da situação, atenção e outras variáveis psicológicas, portanto, como fenômeno multifatorial ${ }^{3}$.

A dor também pode ser classificada em termos temporais como crônica e aguda. A crônica é a perpetuação da dor aguda que, por sua vez, se inicia subitamente, tendo término previsível, acompanhada de reações fisiológicas de alerta, como a midríase, a sudorese aumentada e o aumento das frequiências: cardíaca e respiratória ${ }^{4}$.

O trauma tecidual causado por procedimentos cirúrgicos, e a reação inflamatória decorrente deste processo, podem resultar em condições dolorosas no período pós-opera- tório. Sabe-se que este tipo de dor não tratada pode acarretar diversos prejuízos ao paciente.

A dor pós-operatória é potente desencadeador de resposta ao estresse, ativando o sistema nervoso autonômo o que causa inúmeros efeitos adversos sobre o organismo. A intensidade deste sintoma pode influenciar o desenvolvimento de síndromes dolorosas crônicas pós-cirúrgicas.

A complexidade da fisiologia da dor sugere a necessidade de múltiplos métodos para seu controle adequado, especialmente, quando ela é acentuada, como nos casos de pós-operatório de grandes operações abdominais. As substâncias farmacológicas mais comumente utilizadas para o controle da dor pós-operatória são os opióides, que por sua vez são inibidores de fibras do tipo C. Cerca de $75 \%$ dos receptores espinhais de opióides são encontrados nas imediações pré-sinápticas de fibras-C. Entretanto, foi sugerido que a dor aguda, intensa e "cortante" na parede abdominal, ocasionada pela movimentação do paciente ou pela expectoração no pósoperatório, é pobremente controlada pelos opióides por ser transmitida por fibras nervosas do tipo A-delta. Isso deixa o paciente sem controle adequado da dor impondo a necessidade da utilização de outras técnicas ${ }^{5}$.

Recursos não farmacológicos que se destinam a alíviar a dor pós-operatória, como a acupuntura, a massagem e a crioterapia, vêm sendo utilizados e investigados pela literatura científica. A Eletroestimulação Nervosa Transcutânea (ENT), recurso utilizado pela fisioterapia, vem apresentando bons resultados no controle da dor pós-operatória. Segundo Lampe a ENT representa "um valioso procedimento clínico para o alívio sintomático da dor" ${ }^{\circ}$.

\section{A ENT e seu mecanismo de ação}

A ENT é um recurso não farmacológico para o alívio da dor aguda e crônica, baseado na Teoria das Comportas, proposta por Melzack e Wall ${ }^{7}$. Ela consiste na aplicação de eletrodos percutâneos que emitem uma corrente elétrica com forma de onda tipicamente bifásica, simétrica ou assimétrica,

1. Fisioterapeuta; Doutora em Enfermagem em Saúde Pública pela Escola de Enfermagem de Ribeirão Preto da Universidade de São Paulo; Professora Doutora do Departamento de Biomecânica, Medicina e Reabilitação do Aparelho Locomotor- Curso de Fisioterapia - Faculdade de Medicina de Ribeirão Preto- USP.

2. Fisioterapeuta; Doutoranda em Enfermagem em Saúde Pública pela Escola de Enfermagem de Ribeirão Preto da Universidade de São Paulo; Docente da Universidade de Ribeirão Preto; Universidade Paulista e Faculdades Integradas Fafibe.

Recebido em 05/09/2006

Aceito para publicação em 06/11/2006

Conflito de interesses: nenhum

Fonte de financiamento: nenhuma

Trabalho realizado na Faculdade de Medicina de Ribeirão Preto- USP - Departamento de Biomecânica Medicina e Reabilitação do Aparelho Locomotor - Curso de Fisioterapia. 
com o objetivo de excitar as fibras nervosas, com mínimos efeitos adversos para o paciente ${ }^{6,8}$.

A ENT apresenta, como principal efeito, a analgesia. Apesar de não estar completamente elucidado seu mecanismo fisiológico de ação, é postulado que o estimulo elétrico através da pele inibiria as transmissões dos impulsos dolorosos através da medula espinhal, bem como a liberação de opiácios endógenos, como endorfinas, pelo cérebro ou medula espinhal ${ }^{9,10-12}$.

Estudos laboratoriais têm demonstrado que a ENT diminui a atividade nociceptiva evocada nas células do corno dorsal da medula, quando aplicada em áreas de receptores somáticos, ativando fibras nervosas mielinizadas aferentes de grosso calibre ${ }^{5}$. As pequenas fibras $\mathrm{C}$, não mielinizadas e de condução mais lenta, que por sua vez conduzem os estímulos dolorosos, tornam-se incapazes de transmitir sua mensagem ${ }^{13}$.

\section{Aplicação da ENT no pós-operatório}

O aparelho de Eletroestimulação Nervosa Transcutânea (ENT) é um gerador de pulsos balanceados que envia impulsos elétricos através da pele. É aparelho de corrente de baixa freqüência, do tipo bidirecional e assimétrico, que pode variar de $1 \mathrm{~Hz}$ a $250 \mathrm{~Hz}$, não provocando ionização. Possui dois canais independentes, com quatro eletrodos que foram desenvolvidos para evitar a formação de pontos quentes e, conseqüentemente, lesões cutâneas, do tipo queimadura ${ }^{10}$.

A corrente elétrica gerada pelo aparelho é transmitida através dos fios elétricos até o eletrodo aplicado sobre a pele do paciente. O eletrodo mais comum é o negro, de borracha de silicone, impregnada com carbono. Estes farão contato com a pele através de um elemento de condução intersticial (gel), fixados por película adesiva. Existem também eletrodos do tipo auto-adesivos.

Nas síndromes dolorosas agudas e crônicas normalmente são necessários 25 a 30 minutos de estimulação, variável de duas a três horas, para se obter o efeito analgésico, chegando, muitas vezes, até 12 horas. Pode ser necessária uma nova aplicação.

Existem diversos protocolos para a utilização da ENT, que variam de acordo com a largura e a frequiência de pulso utilizadas. Os mais utilizados são a ENT de alta freqüência, baixa freqüência (ou acupuntura), a breve intensa e a chamada burtz ${ }^{8}$.

As freqüências entre 75 e 100 herts $(\mathrm{Hz})$, chamadas de estimulação convencional ou de alta freqüência, geram impulsos de curta duração e baixa amplitude. A intensidade da corrente é agradável e não gera contração muscular. Este tipo de aplicação é comumente indicado para o controle da dor aguda, como é o caso da dor pós-operatória.

É descrito como inconveniente neste tipo de aplicação, a acomodação neural, ou seja, a diminuição da percepção do estímulo. Isto ocorre pois o nervo torna-se menos excitável à medida que a estimulação é aplicada. Pode-se minimizar esta ocorrência ajustando-se freqüentemente a amplitude da corrente ou modulando o pulso elétrico ${ }^{8}$.

Um dos fatores importantes para se obter bons resultados com a aplicação da ENT, relaciona-se ao posicionamento dos eletrodos. A área selecionada deve estar anatômica ou fisiologicamente relacionada à dor. A região deve ser adequada ao perfeito acoplamento dos eletrodos, o que exclui áreas cobertas por grande quantidade de pêlos e com proeminência óssea.

Parece que grande parte da dor resultante do trauma cirúrgico não advém da incisão cirúrgica em si, mas sim da lesão aos tecidos adjacentes e à musculatura como resultado da retração cirúrgica ${ }^{6}$. Com base nessa hiperalgia secundária recomenda-se que os eletrodos devam ser posicionados duas a três polegadas distantes da incisão cirúrgica ${ }^{6}$.

\section{Contra-Indicações e Efeitos Colaterais}

A ENT é recurso extremamente seguro. Entretanto, em algumas situações, ela não deve ser indicada a fim de evitar complicações hipotéticas. São elas:

- Em portadores de_marcapasso.

- Pessoas cardiopatas.

- Pacientes que apresentam dores sem etiologia definida.

- Epiléticos sem acompanhamento médico.

- Nas gestantes, durante o primeiro trimestre.

A ENT não deve utilizada nas seguintes regiões:

- Nas regiões carotídeas.

- Na boca.

- Na pele com solução de continuidade.

- No abdome_durante a gestação.

- Sobre as pálpebras.

Em alguns casos, podem ocorrer irritação ou erupções cutâneas alérgicas ao redor ou debaixo da colocação dos eletrodos. Havendo persistência dessa irritação, o uso deve ser suspenso.

Avaliação da efetividade da ENT como recurso para o alívio da dor pós-operatória

O uso da ENT como recurso de alívio da dor pósoperatória, pode diminuir o consumo de analgésicos pelos pacientes, bem como reduzir os níveis de dor avaliados por Escalas Visuais Analógicas (EVA) ${ }^{5,14}$.

Outro benefício é a possibilidade da movimentação precoce dos pacientes que é facilitada pela redução da dor propiciada pelo uso da ENT, utilizada como recurso complementar à analgesia farmacológica ${ }^{5}$. Para a atuação da fisioterapia, isto é de grande importância , uma vez que a permanência do paciente no leito por longos períodos, acarreta complicações que retardam a sua recuperação.

Chen et al. compararam a utilização da ENT convencional utilizada na região peri-incisional, com a ENT utilizada sobre pontos de acupuntura no alívio da dor pós-histerectomia ou miomectomia. Neste estudo, foram incluídas 100 mulheres randomicamente distribuídas em quatro grupos: no Grupo 1 o aparelho estava desligado com os eletrodos posicionados em pontos de acupuntura; no Grupo 2 o aparelho estava ligado com o eletrodo posicionado nos ombros, fora de pontos de acupuntura; o $\mathrm{G}$ rupo 3 utilizou o aparelho ligado com os ele- 
trodos posicionados na região peri-incisional; no Grupo 4 o aparelho estava ligado com os eletrodos posicionados sobre os pontos de acupuntura. A ENT foi ajustada intercalando baixa e alta frequiência, que alternava 2 e 100 hertz $^{15}$.

Foram avaliadas, em todos os grupos, a requisição e utilização de analgesia por opióides, a intensidade da dor por Escala Visual Analógica em intervalos determinados de tempo, assim como a sedação, fadiga e náusea ${ }^{15}$.

Nas primeiras 24 horas pós-operatória, a requisição de opióides nos Grupos 3 e 4 decresceram 37\% e 39\% respectivamente, comparando com o grupo controle (placebo) e $35 \%$ e $38 \%$ respectivamente comparados ao Grupo 2 . A duração do intervalo de utilização de opióides também diminuiu significativamente nos Grupos 3 e 4 comparados com os Grupos 1 e $2^{15}$.

Os autores concluíram então, que a ENT utilizada na região periincisional e nos pontos de acupuntura são igualmente efetivos na diminuição da dor pós-operatória, bem como na redução da requisição de analgesia ${ }^{15}$.

Bjordal et al. estudaram a efetividade da ENT na diminuição do consumo de analgésicos no pós-operatório de diversos tipos de operações tais como colecistectomia, hemorroidectomia, toracotomia, artroplastia total de joelho e prótese de quadril. Nesta metanálise foram selecionados vinte e um estudos randomizados, controlados e com utilização de grupo placebo perfazendo um total de 1350 pacientes. Em todos os estudos a redução média do consumo de analgésicos após a aplicação da ENT foi em média 26,5\% menor que no grupo placebo. A freqüência média de estímulo utilizada nesses estudos foi 85 hertz $^{14}$.

Rakel e Frantz testaram, a efetividade da ENT como recurso suplementar à analgesia farmacológica sobre a dor ao movimento e ao repouso depois de operação abdominal. Estas autoras avaliaram a intensidade da dor através de Escala Analógica Visual, escala de avaliação da capacidade de deambulação e a capacidade vital através de espirometria, em 33 sujeitos. Neste estudo randomizado e controlado foram constituídos três grupos. Para o primeiro, foi oferecido analgesia farmacológica e a ENT, para o segundo, analgesia farmacológica e ENT placebo e para o terceiro somente analgesia farmacológica ${ }^{5}$.
Os parâmetros de estimulação foram ajustados intercalando a ENT de alta e baixa frequiência. Esta opção baseouse no estudo experimental em gato, realizado por Garrison e Foreman que sugere maior efetividade do alívio da dor pósoperatória, usando estes parâmetros para estimulação. Os eletrodos foram posicionados um de cada lado à incisão abdominal e apertados duas a três polegadas ${ }^{16}$.

A utilização da ENT resultou em redução significante da dor no grupo controle, comparado ao grupo placebo, tanto durante a marcha, como durante a execução de manobras para a avaliação da capacidade vital. No grupo intervenção, a ENT também produziu melhora mais significativa na velocidade da marcha do que o grupo controle e maior distância percorrida do que o grupo controle e o grupo placebo. Entretanto, não foi verificada diferença significativa entre os três grupos em relação à intensidade da dor e avaliação da execução de manobras de capacidade vital ao repouso.

\section{Considerações Finais}

A dor, por ser um fenômeno complexo e multifatorial, exige abordagem multiprofissional, visando melhor qualidade de vida dos pacientes no pós-operatório.

A ENT é um recurso fisioterapêutico para o controle da dor pós-operatória que apresenta vantagens importantes, como baixo custo, fácil aplicabilidade, poucos efeitos colaterais e principalmente boa eficácia relacionada à diminuição da percepção dolorosa e do consumo de analgésicos farmacológicos.

É importante enfatizar a necessidade da utilização da ENT como recurso de alívio da dor inserido em um contexto multiprofissional, avaliando-se conjuntamente com a equipe médica a necessidade de somar a utilização da mesma com outros recursos para que possa melhorar e potencializar a analgesia oferecida aos pacientes no pós-operatório.

Busca-se também conseguir efeito específico relacionado ao alívio da dor pós-operatória e à movimentação precoce, que a literatura sugere ser pobremente controlada por recursos farmacológicos, limitando assim a realização precoce da fisioterapia pós-operatória tão importante na prevenção de diversas complicações.

\begin{abstract}
This study provides an update on the use of Transcutaneous Eletric Nerve Stimulation (TENS) as a non-pharmacological resource of postoperative pain relief. National and international references regarding the use of TENS for the relief of postoperative pain had been selected, highlighting its mechanism of action, parameters of application, contraindications, sideeffect and results. TENS is a non-pharmacological resource that has its mechanism of action based on the gate theory. It consists of a device that emits electric impulses in the skin through electrodes. It is a low cost technique, easily applicable, presents few collateral effects, good effectiveness related to the reduction of the painful perception, allowing early mobilization and reduced pharmacological analgesic consumption. TENS represents another option in the control of postoperative pain and should be inserted in a multiprofessional context (Rev. Col. Bras. Cir. 2007; 34(2): 127-130).
\end{abstract}

Key words: Transcutaneous Electric Nerve Stimulation; Pain, postoperative; Physical therapy (specialty).

\section{REFERÊNCIAS}

1. Beleza ACS. Implantação de uma sistemática de avaliação de dor em um serviço ambulatorial de fisioterapia. [Monografia]. São Carlos: Universidade Federal de São Carlos; 2000.
2. Classification of chronic pain. Descriptions of chronic pain syndromes and definitions of pain terms. Prepared by the International Association for the Study of Pain, Subcommittee on Taxonomy. Pain.1986; Suppl 3: S1-226.

3. Melzack R, WALL PD. Textbook of pain. Edinburg: Churchill Livingstone; 1994. 
4. Soafer B. Dor - Manual prático. Figueiredo JE, trad. Rio de Janeiro: Revinter; 1994.

5. Rakel B, Frantz R. Effectiveness of transcutaneous electrical nerve stimulation on postoperative pain with movement. J Pain. 2003;4(8):455-64.

6. Lampe GE. Estimulação elétrica nervosa transcutânea. In: O’Sullivan SB. Fisioterapia: avaliação e tratamento. $2^{a}$ ed. São Paulo: Manole; 1993. p. 739-60.

7. Melzack R, Wall PD. Pain mechanisms: a new theory. Science. 1965;150(699):971-9.

8. Guirro E. Guirro R. Eletroterapia. In: Guirro E. Guirro R. Fisioterapia dermato - funcional: fundamentos, recursos e patologias.

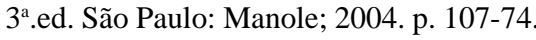

9. van der Spank JT, Cambier DC, De Paepe HM, Danneels LA, Witvrouw EE, Beerens L. Pain relief in labour by transcutaneous electrical nerve stimulation (TENS). Arch Gynecol Obstet. 2000;264(3):131-6.

10. Ferreira CHJ, Payno SMA. Eletroestimulação nervosa transcutânea como recurso de alivio da dor no trabalho de parto. Femina. 2002;30(2):83-6.

11. Frapton V. Eletroestimulação nervosa transcutânea. In: Kitchen S, Bazin, S. Eletroterapia de Clayton. $10^{\mathrm{a}}$ ed. São Paulo: Manole; 1998. p. 276-94.

12. Breit R, Van der Wall H. Transcutaneous electrical nerve stimulation for postoperative pain relief after total knee arthroplasty. J Arthroplasty. 2004;19(1):45-8.

13. Ferreira CHJ. Avaliação de dois recursos não-farmacológicos de alívio para o alívio da dor durante o trabalho de parto. [Dissertação]. Ribeirão Preto: Universidade de São Paulo; 2004.
14. Bjordal JM, Johnson AEL. Transcutaneous electrical nerve stimulation (TENS) can reduce postoperative analgesic consumption. A meta-analysis with assessment of optimal treatment parameters for postoperative. Eur J Pain. 2003;7(2):181-8.

15. Chen L, Tang J, White PF, Sloninsky A, Wender RH, Naruse R, Kariger R. The effect of location of transcutaneous electrical nerve stimulation on postoperative opioid analgesic requirement: acupoint versus nonacupoint stimulation. Anesth Analg. 1998;87(5):129-34.

16. Garrison DW, Foreman RD. Decreased activity of spontaneous and noxiously evoked dorsal horn cells during transcutaneous electrical nerve stimulation (TENS). Pain. 1994;58(3):309-15.

Como citar este artigo:

Ferreira CHJ, Beleza ACS. Abordagem fisioterapêutica na dor pósoperatória: a eletroestimulação nervosa transcutânea (ENT). Rev Col Bras Cir. [periódico na Internet] 2007; 34(2). Disponível em URL: http://www.scielo.br/rcbcb

Endereço para correspondência:

Cristine Homsi Jorge Ferreira

Dep. Biomecânica, Medicina e Reabilitação do Aparelho Locomotor Faculdade de Medicina de Ribeirão Preto - FMRP

Universidade de São Paulo - USP

Curso de Fisioterapia - Prédio Central

Av. Bandeirantes, 3900

14049-900 Ribeirão Preto-SP

Fone: (16) 6024413/6024415 Fax: 166330336

e-mail: crishjferreira@yahoo.com.br 